\title{
CANEUS2006-11023
}

\section{TOWARDS MULTIFUNCTIONAL CHARACTERISTICS OF EMBEDDED STRUCTURES WITH CARBON NANOTUBE YARNS}

\author{
Corey D. Hernandez \\ Swales Aerospace \\ Hampton, VA 23681 USA \\ c.d.hernandez@larc.nasa.gov
}

\author{
Thomas S. Gates and Seun K. Kahng* \\ NASA Langley Research Center \\ Hampton, VA 23681 USA \\ Thomas.S.Gates@nasa.gov \\ *P.O.C.: Seun.K.Kahng@nasa.gov
}

\begin{abstract}
This paper presents recent results on research of achieving multifunctional structures utilizing Carbon Nanotube (CNT) yarns. The investigation centers on creating composite structures with CNT yarns to simultaneously achieve increases in mechanical strength and the ability to sense strain.

The CNT yarns used in our experiments are of the single-ply and two-ply variety with the single-ply yarns having diameters on the order of $10-20 \mu \mathrm{m}$. The yarns are embedded in silicon rubber and polyurethane test specimens. Mechanical tests show an increase in modulus of elasticity, with an additional weight increase of far less than one-percent. Sensing characteristics of the yarns are investigated on stainless steel test beams in an electrical bridge configuration, and are observed to have a strain sensitivity of $0.7 \mathrm{mV} / \mathrm{V} / 1000$ micro-strain. Also reported are measurements of the average strain distribution along the direction of the CNT yarns on square silicon rubber membranes.
\end{abstract}

To expand the performance characteristics of the next generation of revolutionary aerospace design concepts researchers will rely on, among other technologies, selective use of light-weight, multifunctional materials. This trend has put an increased emphasis on the ability to find material candidates that meet strict aerospace vehicle requirements [1]. Examples of recent research programs related to the presented work include research on selective mechanical reinforcement and development of integrated health monitoring of aerospace vehicles and space structures. Possible applications of these technologies include inflatable structures, manned space suits and manned space habitats.

CNT yarns are viable candidates for both multifunctionality and sensing because of their inherent mechanical strength, and light weight characteristics and high degree of thermal and electrical conductivity.

The use of CNT yarns in part is motivated by the high strength and mechanical properties found in the individual carbon nanotubes that comprise the yarn bundle [3]. These mechanical properties are matched or bettered by a few other technologies such as carbon nanotube fibers [4-8].

The ability to manufacture CNT yarns is a relatively recent development in nanotechnology with the first published reports of yarns similar to the ones utilized in this paper dating back to 2002 . The ability to increase yarn strength through spinning dates back to 2004 [2,9].

The objective of the work presented is to present results on initial steps taken at NASA Langley Research Center to incorporate CNT yarns into composite structures that provide the multifunctionality of mechanical strength enhancement and strain sensing.

Four individual experiments are reported towards the development of multifunctional structures utilizing CNT yarns. The experiments presented are: 1) yarn resistance dependence on injection current and temperature, 2) tensile strength tests on thin composite samples reinforced with the CNT yarns, 3) strain sensitivity of the CNT yarns using a cantilever test beam, and 4) average strain measurements along the length of a CNT yarn embedded on the surface of a membrane.

The work presented here utilizes CNT yarns provided by Ray Baughman's research team at the NanoTech Institute and Department of Chemistry at the University of Texas at Dallas [2, 10-13]. The spun yarns are produced by drawing and spinning the yarns out of highly-aligned MWCNT (Multi-Walled Carbon 
Nanotubes) forests grown by the Chemical Vapor Deposition process (CVD) using an iron catalyst coated on a silicon substrate. The yarns are drawn out of the forest, which stands approximately $200 \mu \mathrm{m}$ high off of the substrate and covers a surface area on the scale of centimeters, using a cylindrical spindle rotating at 2000 rpm [2].

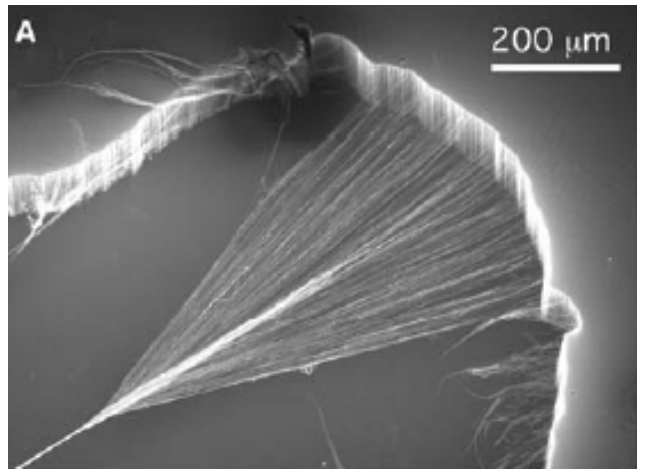

Figure 1: SEM image of nanotubes in the process of being drawn out of the nanotube forest and spun into yarn [2].

Baughman's group has demonstrated the ability to draw yarns to lengths of over 10-meters with diameters on the order of 10 to $40 \mu \mathrm{m}$ and the ability to manufacture single, 2- and 4-ply yarns. The group has shown individual yarns to have tensile strengths greater than $460 \mathrm{MPa}$ and effective yarn tensile moduli greater than 20GPa. Furthermore they have found the yarns to have a four probe electrical conductivity of $300 \mathrm{~S} / \mathrm{cm}$ at room temperature and a negative temperature dependence of approximately $0.1 \%$ per degree Celsius. They have also been shown to retain their strength and flexibility after heating in air to $+460^{\circ} \mathrm{C}$ and immersed in liquid nitrogen at $-196^{\circ} \mathrm{C}$ [2].

The most interesting physical property from a sensing perspective is the unusually high Poisson's ratio observed by Baughman et al that ranges from 2.0 to 2.7 for multiwalled CNT singles yarn and from 3.3 to 4.2 for the two-ply yarn. Ordinary solids, including materials used in typical strain gages, have typical Poisson's ratios on the order of $\sim 0.3$. With strain sensitivity directly related to two-times the Poisson's ratio, the potential use of yarns as imbedded strain gages exists [2].

In all, the resultant physical properties of these yarns are meters long in length with high electrical conductivity, high strength to weight ratios, and the potential to sense strain and temperature.

Individual CNTs have been shown to have sensitivity to temperature and recently Baughman et al. have shown the same for CNT yarns [2].

To characterize the temperature dependence of the CNT yarns researchers suspended a yarn between an electrode pair and exposed the setup to ambient air in a thermal chamber. The chamber's temperature is varied between -60 degree $C$ and +130 degree $C$, and the CNT yarns are injected with currents of $1 \mu \mathrm{A}, 5 \mu \mathrm{A}, 100 \mu \mathrm{A}$, $500 \mu \mathrm{A}$ and $1000 \mu \mathrm{A}$ at prescribed temperatures. Typical results of this experiment are illustrated in Figure 2a-b where we found the yarns to have a negative temperature dependence of approximately $0.07 \%$ per degree Celsius. These results are in line with the $0.10 \%$ results reported by Baughman et al. [2].

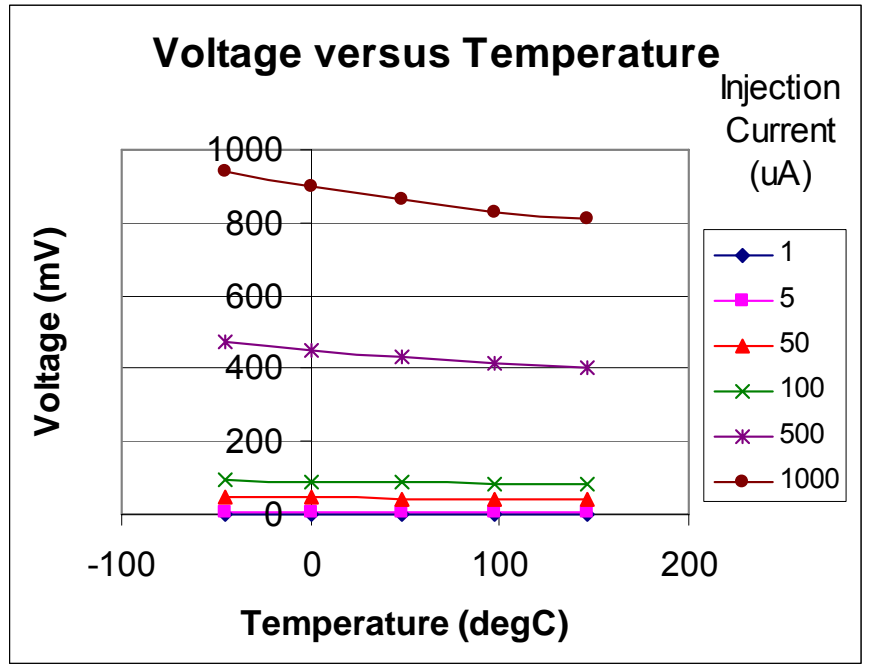

Figure 2-a: Typical voltage across the CNT yarns with varying injection currents as a function of temperature.

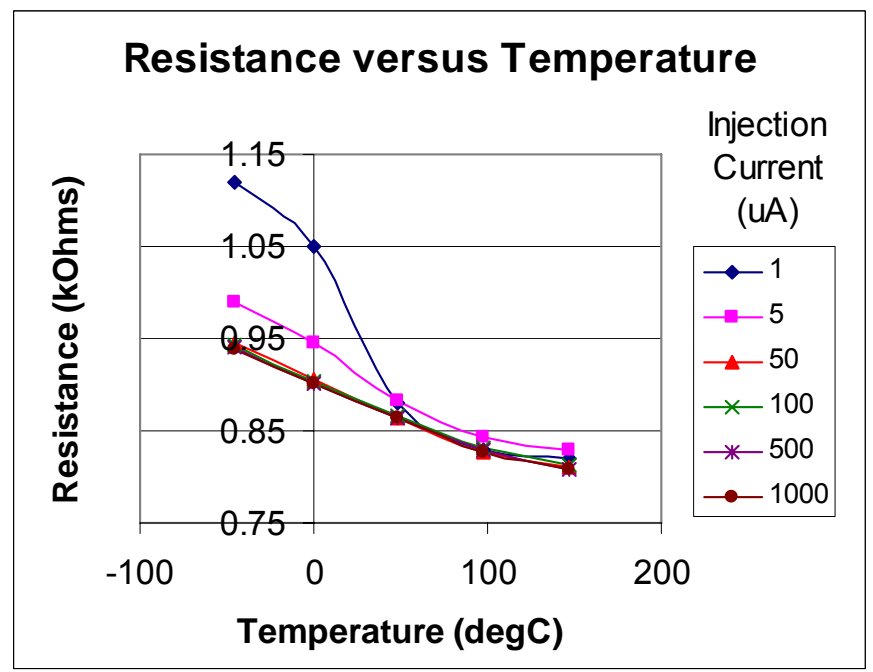

Figure 2-b: Resistance values of the yarns with injection currents as a function of temperature.

With the injection current values used, $(1 \mu \mathrm{A}, 5 \mu \mathrm{A}$, $100 \mu \mathrm{A}, 500 \mu \mathrm{A}$, and $1000 \mu \mathrm{A}$ ) estimated current densities range from 0.3184 to $159.2 \mathrm{Amp} / \mathrm{sq}-\mathrm{cm}$. As Figure 2 indicates, as the injection current increases the resistance values of the yarn tend lower, and the temperature coefficient of resistance increases. Additionally, at higher temperatures, the resistance values tend to converge to that of the higher injection current.

In order to evaluate mechanical tensile strength, CNT yarns are embedded into silicon rubber and polyurethane samples approximately 0.2 to $0.8 \mathrm{~mm}$ thick 
with gage lengths of $25.4 \mathrm{~mm}$. The samples are cut into a typical dog-bone geometry using a laser ablation method to ensure a uniformly cut and continuous edge (Figure 3). The CNT yarns embedded in the polyurethane extends the entire length between both grip ends. The actual length and width of the test samples are $25.4 \mathrm{~mm}$ and $12.7 \mathrm{~mm}$, respectively. The samples are subjected to a uniaxial tensile test on a electro-mechanical test stand at room temperature under a constant applied displacement rate. Axial strain was measured with a non-contacting laser extensometer and load was measured with the test machine load cell. Stress was computed on the average gage cross-section measured prior to the test and strength was defined as a complete loss of load carrying capacity during loading. Longitudinal elastic modulus was determined from the slope of the resultant stress-strain curve. An example of a typical result from this experiment is shown in Figure 4. Representative data of the experimentally determined tensile moduli are compared in Table 1.

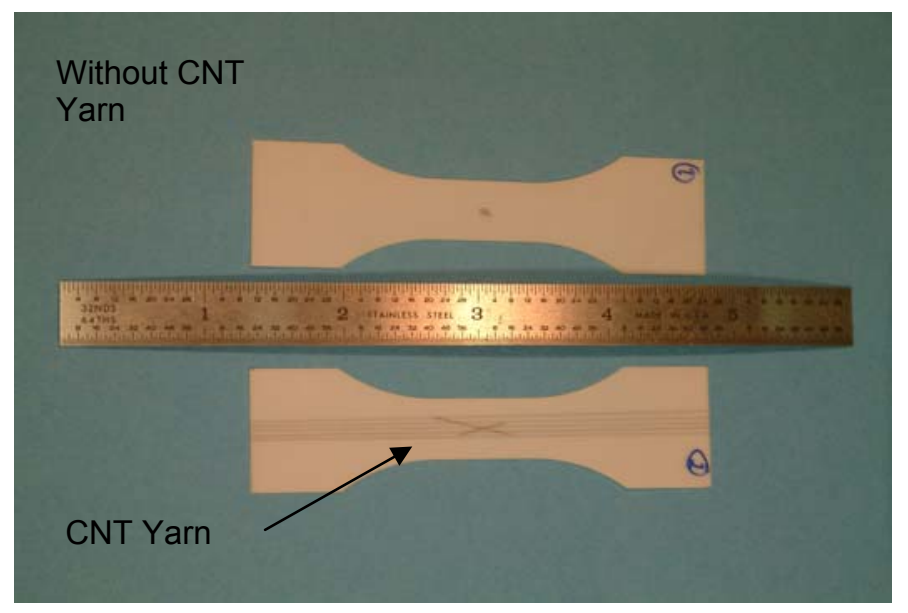

Figure 3: Samples for tensile strength test, top one does not have CNT yarns embedded and the bottom one does.

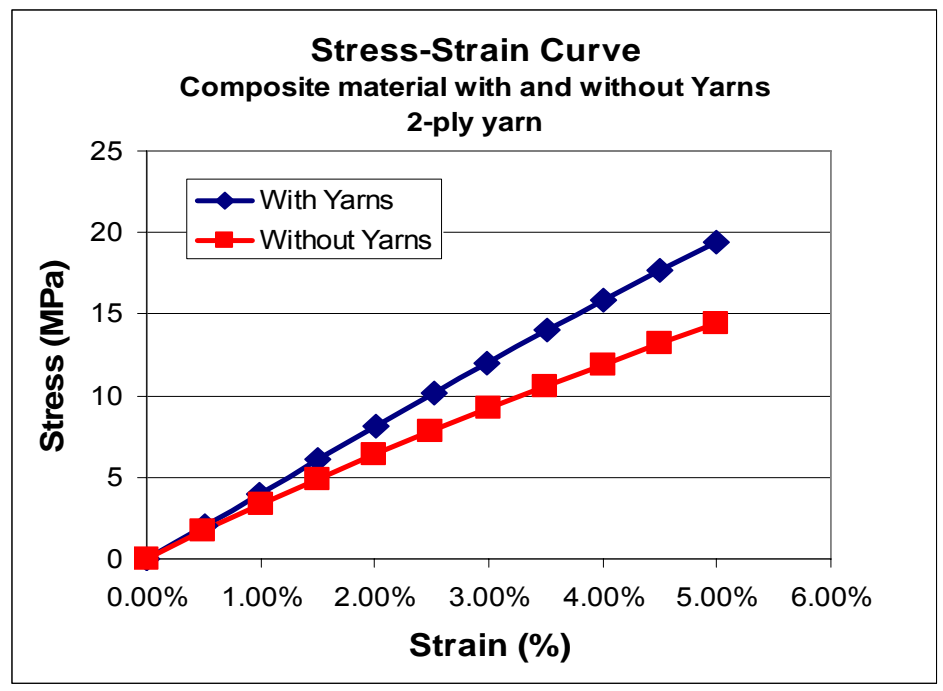

Figure 4: Typical Stress-Strain results of CNT yarn embedded versus non-embedded test samples.
Table 1: Tabular results from mechanical strength test comparing CNT embedded polyurethane samples to the non-embedded test samples

\begin{tabular}{|c|c|c|c|c|}
\hline Sample \# & 1 & 2 & 3 & 4 \\
\hline $\begin{array}{c}\text { Thickness } \\
(\mathrm{mm})\end{array}$ & 0.69 & 0.81 & 0.58 & 0.508 \\
\hline Material & $\begin{array}{c}\text { Clear } \\
\text { Flex 95 }\end{array}$ & $\begin{array}{c}\text { Clear } \\
\text { Flex 95 }\end{array}$ & $\begin{array}{c}\text { Ren } \\
6405\end{array}$ & $\begin{array}{c}\text { Ren } \\
6405\end{array}$ \\
\hline $\begin{array}{c}\text { Modulus } \\
\text { w/o Yarns } \\
\text { (MPa) }\end{array}$ & 14.96 & 5.4 & 652 & 582 \\
\hline $\begin{array}{c}\text { Modulus } \\
\text { w/ Yarns } \\
\text { (MPa) }\end{array}$ & 16.23 & 7.95 & 813 & 877 \\
\hline \# of Yarns & 11 & 6 & 10 & 10 \\
\hline $\begin{array}{c}\text { Increase } \\
\text { in } \\
\text { Modulus } \\
\text { (Factor) }\end{array}$ & 1.23 & 1.29 & 1.37 & 1.61 \\
\hline
\end{tabular}

Preliminary results indicate that composite materials with embedded CNT yarns have an increase modulus of elasticity of $20 \%$ to $60 \%$ compared to baseline polyurethane data. Typical data of two polyurethanes with different modulus of elasticities are illustrated in Table 1.

To evaluate the applicability of CNT yarns to strain sensing, CNT yarns are embedded on the surface of a stainless steel cantilever test beam utilizing polyurethane as an adhesive. The beam under test is $20.32 \mathrm{~cm}$ long, $2.54 \mathrm{~cm}$ wide, and $0.318 \mathrm{~cm}$ thick stainless steel (17$4 \mathrm{PH})$. The yarn is placed in a Wheatstone bridge electrical configuration while the cantilever is loaded in simple bending. The bridge output voltage is then recorded and compared against standard metal foil strain gages located on the opposite side of the test beam. The resultant output voltages, normalized by that of 100 micro-strain, are shown in Figure 6 for input voltages varying from $0.3,0.5,0.7$, and 1.0 volt.

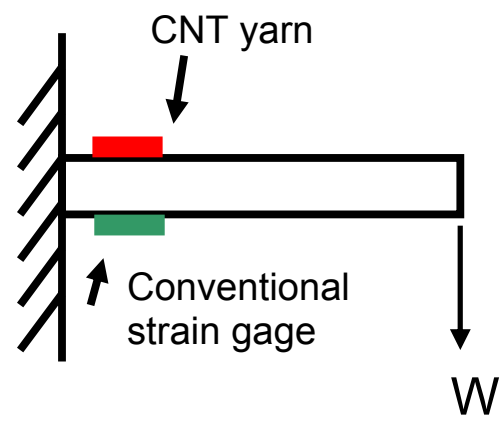

Figure 5: Diagram outlining the experimental setup used to measure the response of the CNT yarns to applied strain on a cantilever beam. 


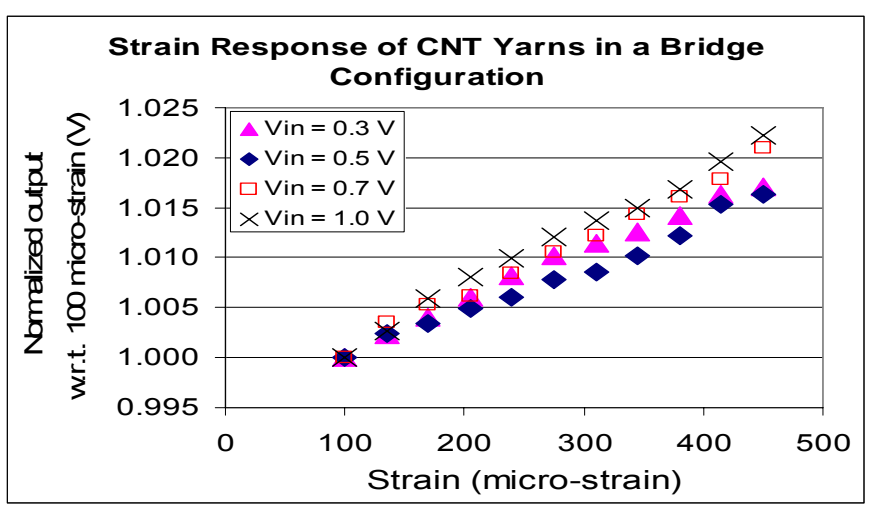

Figure 6: Response of 1/4 Wheatstone bridge with CNT yarns as a function of induced strain on a stainless steel cantilever beam as measured by conventional strain gages.

The results shown in Figure 6 show a linear correlation between the strain measured by the standard strain gages and the normalized output of the electrical bridge (normalized to the voltage output at 100 micro-strain).

To further investigate the strain sensing properties of the yarns, a $16 \mathrm{~cm}$ by $16 \mathrm{~cm}$ Silicon rubber membrane is fabricated with five CNT yarns embedded near the surface. The yarns are evenly spaced and run across the entire length of the membrane. The square, flexible membrane is fixed on all sides while the center is unsupported. A point deflection is applied to the center of the membrane while a constant current of $5 \mu \mathrm{A}$ is applied through each yarn. The center deflection is measured using a high accuracy laser displacement measuring device. The changes in yarn resistance versus center deflection are recorded.

The percentage change in resistance is then compared to the theoretically derived values of average strain determined using finite element software. The comparative results are shown in Figure 7. The finite element analysis is conducted using fixed edges with material properties similar to those of the polyurethane used in the test. The values presented are derived from averaging nodal strains across the path of the yarn.

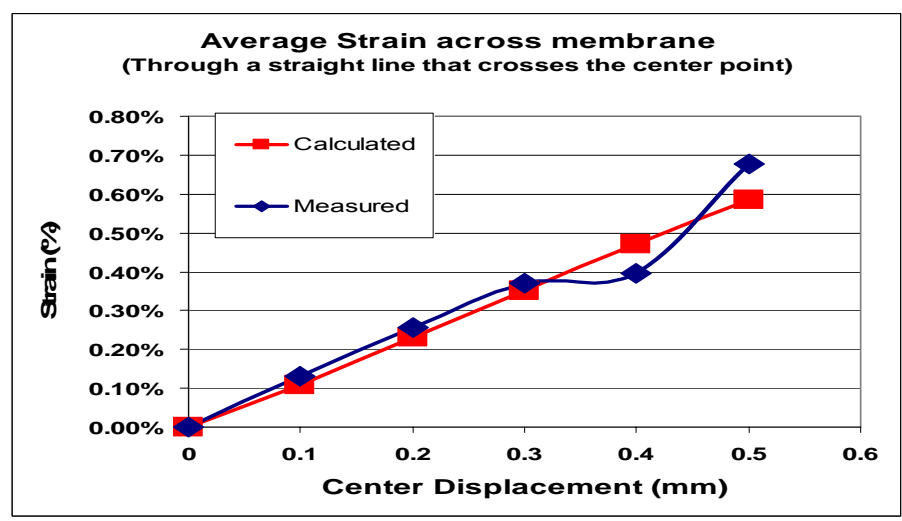

Figure 7: Comparison of theoretical and measured values of strain as a function of the displacement of the center of the composite membrane. The theoretical values are derived from a finite element analysis of the membrane.
For lower strain values, initial results show relative agreement between the calculated and measured values. The results shown are typical results demonstrated across multiple experiments.

From a sensing perspective, the temperature characterization data agreed with previously published data and demonstrated the yarns ability to sense temperature. Additionally, the results of the cantilever test beam and thin membrane experiment demonstrated the first steps towards using the yarns as a strain sensor. Furthermore, the strain and temperature tests also showed the response of the yarns to be a function of injection current.

The above was accomplished while simultaneously demonstrating the yarns ability to enhance the mechanical strength of given materials. This is the first step towards showing that for a slight weight penalty a significant increase in the modulus of a membrane can be achieved using carbon nanotube yarns.

\section{References}

1. National Aeronautics and Space Administration 2005 Strategic Plan

2. M. Zhang, K. Atkinson, R.H. Baughman, Science 306, 1358 (2004)

3. R.H. Baughman, A.A. Zakhidov, W.A. de Heer, Science 297, 787 (2002)

4. P. Miaudet, et al., Nano Letters, 5, No. 11, 2212 (2005)

5. Li, Y et al., Science 2004. 304, 276

6. L.M. Ericson et al., Science 305, 1447 (2000)

7. Dalton, et al., Nature, 2003, 423, 703

8. B. Vigolo et al,. Science 290, 1331 (2000)

9. K. Jiang, Q. Li, S. Fan, Nature 419, 801 (2002)

10. Winters, J., Mechanical Engineering Magazine, 128 No. 4, 2006

11. R.H. Baughman, Science 308, 63 (2005)

12. Baughman, R.H., Science, $290,1310,(2000)$

13. M. Zhang, et al., Science 309, 1215 (2005) 